\title{
THE FILLING DISKS OF AN ALGEBROID FUNCTION IN THE UNIT DISK
}

\author{
ZU-XING XUAN
}

(Received 16 July 2009)

\begin{abstract}
Using potential theory, we prove the existence of filling disks of an algebroid function of finite order defined in the unit disk.
\end{abstract}

2000 Mathematics subject classification: primary 30D35.

Keywords and phrases: algebroid function, filling disks, covering surface, order.

\section{Introduction and main result}

The value distribution theory of meromorphic functions, due to Nevanlinna (see [1] for standard references), was extended to the corresponding theory of algebroid functions by Selberg [2], Ullrich [4] and Valiron [5] around 1930. Investigating the filling disks of an algebroid function is a basic problem in value distribution theory. For an algebroid function defined on the $z$-plane, the existence of its filling disks was proved by Sun in [3]. Compared with the case of $\mathbb{C}$, it is interesting to investigate algebroid functions defined in the unit disk, and there are some essential differences between these two cases. Thus we raise the following question.

QUESTION 1.1. Is there any similar result for the algebroid functions defined in the unit disk?

This paper investigates this problem, and we confine our attention to algebroid functions defined in the unit disk $\Delta=\{z:|z|<1\}$.

Let $w=w(z), z \in \Delta$, be the $v$-valued algebroid function defined by the irreducible equation

$$
A_{v}(z) w^{v}+A_{v-1}(z) w^{\nu-1}+\cdots+A_{0}(z)=0,
$$

where $A_{v}(z), \ldots, A_{0}(z)$ are entire functions with no common zeros. The singlevalued domain of definition of $w(z)$ is a $v$-valued covering of the $z$-plane, a Riemann surface, denoted by $\widetilde{R}_{z}$. A point in $\widetilde{R}_{z}$, whose projection in the $z$-plane

The work is supported by NSF of China (No. 10871108, 10926049).

(C) 2010 Australian Mathematical Publishing Association Inc. 0004-9727/2010 \$16.00 
is $z$, is denoted by $\widetilde{z}$. The part of $\widetilde{R}_{z}$ that covers the disk $\{z:|z|<r\}$ is denoted by $|\widetilde{z}|<r$.

Define

$$
S(r, w)=\frac{1}{\pi} \iint_{|\widetilde{z}| \leq r}\left[\frac{\left|w^{\prime}(z)\right|}{1+|w(z)|^{2}}\right]^{2} d \omega .
$$

Then $S(r, w)$ is called the mean covering number of $|\widetilde{z}| \leq r$ into the $w$-sphere under the mapping $w=w(z)$ and $S(r, w)$ is a conformal invariant. Let $n(r, a)$ be the number of zeros of $w(z)-a$, counted according to their multiplicities in $|\widetilde{z}| \leq r$, and let $\bar{n}(E, w=\alpha)$ denote the number of zeros of $w(z)=\alpha$ in $E$, each zero being counted only once.

Let

$$
N(r, a)=\frac{1}{v} \int_{0}^{r} \frac{n(t, a)-n(0, a)}{t} d t+\frac{n(0, a)}{v} \log r
$$

and

$$
m(r, a)=\frac{1}{2 \pi v} \int_{|\widetilde{z}|=r} \sum_{j=1}^{v} \log ^{+}\left|\frac{1}{w_{j}\left(r e^{i \theta}\right)-a}\right| d \theta, \quad z=r e^{i \theta},
$$

where $|\widetilde{z}|=r$ is the boundary of $|\widetilde{z}| \leq r$. The characteristic function of $w(z)$ is defined by

$$
T(r, w)=\frac{1}{v} \int_{0}^{r} \frac{S(t, w)}{t} d t .
$$

In view of [5],

$$
T(r, w)=m(r, w)+N(r, \infty)+O(1) .
$$

The order of the algebroid function $w(z)$ is defined by

$$
\rho=\limsup _{r \rightarrow 1-} \frac{\log T(r, w)}{\log \frac{1}{1-r}} .
$$

In this paper we assume that $0<\rho<+\infty, V$ is the $w$-sphere, and $C$ is a constant whose value depends on the context. Let $n\left(r, \widetilde{R}_{z}\right)$ be the number of the branch points of $\widetilde{R}_{z}$ in $|\widetilde{z}| \leq r$, counted with the branch order. Write

$$
N\left(r, \widetilde{R}_{z}\right)=\frac{1}{v} \int_{0}^{r} \frac{n\left(t, \widetilde{R}_{z}\right)-n\left(0, \widetilde{R}_{z}\right)}{t} d t+\frac{n\left(0, \widetilde{R}_{z}\right)}{v} \log r .
$$

In view of [4], we know that

$$
N\left(r, \widetilde{R}_{z}\right) \leq 2(v-1) T(r, w)+O(1)
$$

Valiron was the first to introduce the concept of a proximate order $\rho(1 /(1-r))$ for a meromorphic function $w(z)$ with finite positive order, and

$$
U\left(\frac{1}{1-r}\right)=\left(\frac{1}{1-r}\right)^{\rho(1 /(1-r))}
$$


is called the type function of $w(z)$ or $T(r, w)$. Then $\rho(1 /(1-r))$ is nondecreasing, piecewise continuous and differentiable, and

$$
\begin{gathered}
\lim _{r \rightarrow 1-} \rho\left(\frac{1}{1-r}\right)=\rho, \\
\lim _{r \rightarrow 1-} \frac{U\left(\frac{k}{1-r}\right)}{U\left(\frac{1}{1-r}\right)}=k^{\rho} \quad(k \text { is a positive constant }), \\
\lim _{r \rightarrow 1-} \frac{T(r, w)}{U\left(\frac{1}{1-r}\right)}=1, \\
\lim _{r \rightarrow 1-} \frac{\left(\frac{1}{1-r}\right)^{\rho-\varepsilon}}{U\left(\frac{1}{1-r}\right)}=0, \quad 0<\varepsilon<\rho .
\end{gathered}
$$

For an algebroid function $w(z)$ of finite positive order, we can apply the same method to get its type function $U(1 /(1-r))$.

In this paper, we give a positive answer to the above question by proving the following theorem.

THEOREM 1.2. Suppose that $w(z)$ is the $\nu$-valued algebroid function of finite order $\rho$ in $|z|<1$ defined by (1.1). Then there exists a sequence of disks

$$
\Gamma_{n}:\left\{\left|z-z_{n}\right|<r_{n} \sigma_{n}\right\}, \quad n=1,2, \ldots,
$$

where

$$
z_{n}=r_{n} e^{i \theta_{n}}, \quad \lim _{n \rightarrow \infty} r_{n}=1, \quad \sigma_{n}>0 \quad \text { and } \quad \lim _{n \rightarrow \infty} \sigma_{n}=0,
$$

such that for each $n, w(z)$ takes every complex number at least $(1-r)^{-\rho+\varepsilon_{n}}$ times in $\Gamma_{n} \cap \triangle$, except for those complex numbers contained in the union of $2 v$ spherical disks each with radius $\left(1-r_{n}\right)^{\rho / 40}$, where $\lim _{n \rightarrow+\infty} \varepsilon_{n}=0, \Delta=\{z:|z|<1\}$.

The disks with the above property are called filling disks.

\section{Two lemmas}

LEMMA 2.1 [3]. Suppose that $w(z)$ is the $v$-valued algebroid function in $\{z:|z|<R\}$ defined by (1.1), and $a_{1}, a_{2}, a_{3}, \ldots, a_{q}(q \geq 3)$ are distinct points given arbitrarily in the $w$-sphere, and the spherical distance of any two points is no smaller than $\delta \in\left(0, \frac{1}{2}\right)$. Then for any $r \in(0, R)$,

$$
(q-2) S(r, w) \leq \sum_{j=1}^{q} \bar{n}\left(R, a_{j}\right)+n\left(R, \widetilde{R}_{z}\right)+\frac{2^{15} \pi^{40} \nu R}{(R-r) \delta^{38}} .
$$

Combining potential theory with Lemma 2.1, we prove Lemma 2.2, which is crucial to our theorem.

LEMMA 2.2. Suppose that $w(z)$ is the $v$-valued algebroid function of finite order $\rho$ satisfying $0<\rho<+\infty$ in $|z|<1$ defined by (1.1). For any $\varepsilon \in(0, \rho), 0<R<1$, 
there exists $a_{0} \in\left(\frac{1}{2}, 1\right)$, such that for any $a \in\left(a_{0}, 1\right)$, we can write

$$
\begin{gathered}
r_{n}=1-a^{n}, \quad m=\left\lfloor\frac{2 \pi}{1-a}\right\rfloor, \quad \theta_{q}=\frac{2 \pi(q+1)}{m}, \\
\Omega_{p q}=\left\{1-a^{p} \leq|z|<1-a^{p+2}\right\} \cap\left\{\left|\arg z-\theta_{q}\right| \leq \frac{2 \pi}{m}\right\}
\end{gathered}
$$

where $p=1,2,3, \ldots, q=0,1,2, \ldots, m-1$ and $\lfloor x\rfloor$ stands for the integer part of $x$.

Then, among the pairs $p, q$, there exists at least one pair $p_{0}, q_{0}$ such that $1-a^{p_{0}}>$ $R$, and in $\Omega_{p_{0} q_{0}}$ the function $w(z)$ takes every complex number at least $a^{-p_{0}(\rho-\varepsilon)}$ times (each zero being counted only once), except for those complex numbers contained in the union of $2 v$ spherical disks each with radius $\delta=a^{p_{0} \rho / 40}$.

PROOF. We argue by contradiction. If the result is false, then there exists a sequence $\left\{a_{i}\right\}_{i=1}^{\infty}\left(0<a_{i}<1\right)$, where $\lim _{n \rightarrow \infty} a_{i}=1$. For any $a \in\left\{a_{i}\right\}$, any

$$
p>P=\log (1-R) / \log a \quad \text { and } \quad q \in\{0,1,2, \ldots, m-1\},
$$

there exist $2 v+1$ complex numbers that satisfy the condition that the spherical distance of any two of the points is no smaller than $\delta=a^{p \rho / 40}$. Let $\alpha_{j}=\alpha_{j}(p, q)$ for $j=1, \ldots, 2 v+1$. For any $p, q$ mentioned above,

$$
\bar{n}\left(\Omega_{p q}, w=\alpha_{j}\right)<a^{-p(\rho-\varepsilon)} .
$$

For any $r>R$, let $T=\lfloor\log (1-r) / \log a\rfloor$. Then $1-a^{T} \leq r<1-a^{T+1}$.

For positive integers $N$ and $M$, set

$$
\begin{gathered}
b=a^{1 / M} \in(0,1), \quad \gamma_{p t}=1-b^{M p+t}, \quad t=0,1,2, \ldots, M-1, \\
L_{p t}=\left\{\gamma_{p t} \leq|z|<\gamma_{p, t+1}\right\}, \\
\theta_{q j}=\frac{2 \pi q}{m}+\frac{2 \pi j}{N m}, \\
\triangle_{q j}=\left\{z:|z|<1-a^{T}, \theta_{q j} \leq \arg z<\theta_{q, j+1}\right\} .
\end{gathered}
$$

Then

$$
\left\{1-a \leq|z|<1-a^{T}\right\}=\bigcup_{t=0}^{M-1} \bigcup_{p=1}^{T-1} L_{p t}
$$

and

$$
\left\{|z|<1-a^{T}\right\}=\bigcup_{j=0}^{N-1} \bigcup_{q=0}^{m-1} \triangle_{q j} .
$$


Thus there exist $t_{0}, j_{0}$ which are related to $T$. We can assume that $t_{0}=0, j_{0}=0$, such that

$$
\begin{aligned}
& \sum_{p=1}^{T-1} n\left(L_{p 0}, \widetilde{R}_{z}\right) \leq \frac{1}{M} n\left(1-a^{T}, \widetilde{R}_{z}\right), \\
& \sum_{q=0}^{m-1} n\left(\triangle_{q 0}, \widetilde{R}_{z}\right) \leq \frac{1}{N} n\left(1-a^{T}, \widetilde{R}_{z}\right) .
\end{aligned}
$$

Set

$$
\begin{gathered}
\Omega_{p q}^{0}=\left\{1-\frac{b^{M p}+b^{M p+1}}{2} \leq|z|<1-\frac{b^{M p+M}+b^{M p+M+1}}{2}\right\} \\
\cap\left\{\frac{\theta_{q 0}+\theta_{q 1}}{2} \leq \arg z<\frac{\theta_{q+1,0}+\theta_{q+1,1}}{2}\right\}
\end{gathered}
$$

and

$$
\bar{\Omega}_{p q}=\left\{1-b^{M p} \leq|z|<1-b^{M p+M+1}\right\} \cap\left\{\theta_{q 0} \leq \arg z<\theta_{q+1,1}\right\} .
$$

Then

$$
\Omega_{p q}^{0} \subset \bar{\Omega}_{p q} \subset \Omega_{p q} .
$$

Since $\left\{\bar{\Omega}_{p q}\right\}_{p, q}$ covers $\bigcup_{p=1}^{T-1} L_{p 0}$ and $\bigcup_{q=0}^{m-1} \triangle_{q 0}$ twice at most, we obtain

$$
\sum_{p=1}^{T-1} \sum_{q=0}^{m-1} n\left(\bar{\Omega}_{p q}, \widetilde{R}_{z}\right) \leq\left(1+\frac{1}{M}+\frac{1}{N}\right) n\left(1-a^{T}, \widetilde{R}_{z}\right)
$$

Obviously, each $\bar{\Omega}_{p q}$ can be mapped conformally to the unit disk $|\zeta|<1$ such that the center of $\bar{\Omega}_{p q}$ is mapped to $\zeta=0$, and the image of $\Omega_{p q}^{0}$ is contained in the disk $|\zeta|<\eta(<1)$. Since all $\bar{\Omega}_{p q}, \Omega_{p q}^{0}$ are similar, $C$ is independent of $p, q$. Since $S$ is conformally invariant, in view of Lemma 2.1 , we obtain

$$
\begin{aligned}
(2 v-1) & S\left(1-a^{T}, w\right) \\
\leq & (2 v-1) \sum_{p=P+1}^{T-1} \sum_{q=0}^{m-1} S\left(\Omega_{p q}^{0}, w\right)+(2 v-1) S\left(1-a^{p+2}, w\right) \\
\leq & \sum_{p=P+1}^{T-1} \sum_{q=0}^{m-1}\left[\sum_{j=1}^{2 v+1} \bar{n}\left(\bar{\Omega}_{p q}, w=\alpha_{j}\right)+n\left(\bar{\Omega}_{p q}, \widetilde{R}_{z}\right)+\frac{2^{51} \pi^{40} v}{\delta^{38}(1-\eta)}\right] \\
& +(2 v-1) S\left(1-a^{p+2}, w\right) \\
\leq & 3 v T m a^{-T(\rho-\varepsilon)}+\left(1+\frac{1}{M}+\frac{1}{N}\right) n\left(1-a^{T}, \widetilde{R}_{z}\right) \\
& +\frac{3 m v^{2} 2^{51} \pi^{40}}{1-\eta} T\left(a^{-T \rho / 40}\right)^{38}+(2 v-1) S\left(1-a^{p+2}, w\right) .
\end{aligned}
$$


For a sufficiently large integer

Thus we get

$$
T\left(=\left\lfloor\frac{\log (1-r)}{\log a}\right\rfloor\right), \quad r \in\left[1-a^{T}, 1-a^{T+1}\right) .
$$

$$
\begin{aligned}
(2 v-1) S\left(1-\frac{1-r}{a}, w\right) \leq & (2 v-1) S\left(1-a^{T}, w\right) \\
\leq & \left(\frac{1}{1-r}\right)^{\rho-\varepsilon / 2}+\left(1+\frac{1}{M}+\frac{1}{N}\right) n\left(r, \widetilde{R}_{z}\right) \\
& +C\left(\frac{1}{1-r}\right)^{39 \rho / 40}+C
\end{aligned}
$$

where $C$ is a constant.

Claim. For sufficiently fixed large integer $T(=\lfloor\log (1-r) / \log a\rfloor)$,

(i) $k=\left(1-1 /\left(1+a+a^{2}+\cdots+a^{T-1}\right)\right)^{-1}<(2 v+1) /(2 v-2)$,

(ii) $1-t \leq t$, where $t \in\left[1-a^{T}, 1-a^{T+1}\right)$.

To prove this claim, it suffices to prove our result for (i). Let $a>1-3 /(2 v+1)$. Then $2 v+1<3 /(1-a)$. We have $\sum_{i=0}^{\infty} a^{i}=1 /(1-a)<+\infty$, so we can choose a certain sufficiently fixed large integer $T$ such that

$$
2 v+1<3\left(1+a+a^{2}+\cdots+a^{T-1}\right) .
$$

Thus (i) follows.

This yields

$$
\begin{aligned}
\frac{1-\frac{1-t}{a}}{t} & =\frac{1}{a}-\left(\frac{1}{a}-1\right) \frac{1}{t} \\
& \geq \frac{1}{a}-\left(\frac{1}{a}-1\right) \frac{1}{1-a^{T}} \\
& =\frac{1}{a}\left(1-\frac{1}{1+a+\cdots+a^{T-1}}\right)=\frac{1}{a k},
\end{aligned}
$$

where $t \in\left[1-a^{T}, 1-a^{T+1}\right)$. Hence

$$
\begin{aligned}
\int_{1-a^{T}}^{r} \frac{S\left(1-\frac{1-t}{a}, w\right)}{t} d t & =\int_{1-a^{T}}^{r} \frac{S\left(1-\frac{1-t}{a}, w\right)}{1-\frac{1-t}{a}} \frac{1-\frac{1-t}{a}}{t} d t \\
& \geq \frac{1}{a k} \int_{1-a^{T}}^{r} \frac{S\left(1-\frac{1-t}{a}, w\right)}{1-\frac{1-t}{a}} d t \\
& =\frac{1}{a k} \int_{1-a^{T-1}}^{1-(1-r) / a} \frac{S(x, w)}{x} d(1-a(1-x)) \\
& =\frac{1}{k} \int_{1-a^{T-1}}^{1-(1-r) / a} \frac{S(x, w)}{x} d x
\end{aligned}
$$


Next, we deduce that

$$
\begin{aligned}
\int_{1-a^{T}}^{r} \frac{1}{(1-t)^{\rho-\varepsilon / 2} t} d t & \leq \int_{1-a^{T}}^{r} \frac{1}{(1-t)^{\rho+1-\varepsilon / 2}} d t \\
& =-\int_{1-a^{T}}^{r} \frac{1}{(1-t)^{\rho+1-\varepsilon / 2}} d(1-t) \\
& =-\left.\frac{1}{-\rho+\frac{\varepsilon}{2}}(1-t)^{-\rho+\varepsilon / 2}\right|_{1-a^{T}} ^{r} \\
& =\frac{1}{\rho-\frac{\varepsilon}{2}}\left[\frac{1}{(1-r)^{\rho-\varepsilon / 2}}-\frac{1}{\left[1-\left(1-a^{T}\right)\right]^{\rho-\varepsilon / 2}}\right] \\
& \leq \frac{1}{\rho-\frac{\varepsilon}{2}} \frac{1}{(1-r)^{\rho-\varepsilon / 2}},
\end{aligned}
$$

when $r \geq t \geq 1-a^{T}$.

Similarly,

$$
\int_{1-a^{T}}^{r} \frac{1}{(1-t)^{39 \rho / 40} t} d t \leq \frac{1}{\frac{39 \rho}{40}} \frac{1}{(1-r)^{39 \rho / 40}} .
$$

Dividing both sides of (2.2) by $v t$ and integrating from $1-a^{T}$ to $r$,

$$
\begin{aligned}
(2 v-1) & \frac{1}{v} \int_{1-a^{T}}^{r} \frac{S\left(1-\frac{1-t}{a}, w\right)}{t} d t \\
\leq & \frac{1}{v} \int_{1-a^{T}}^{r} \frac{1}{(1-t)^{\rho-\varepsilon / 2} t} d t+\left(1+\frac{1}{M}+\frac{1}{N}\right) \\
& \times\left[\frac{1}{v} \int_{1-a^{T}}^{r} \frac{n\left(t, \widetilde{R}_{z}\right)-n\left(0, \widetilde{R}_{z}\right)}{t} d t+\frac{n\left(0, \widetilde{R}_{z}\right)}{v} \log r\right. \\
& \left.-\frac{n\left(0, \widetilde{R}_{z}\right)}{v} \log \left(1-a^{T}\right)\right]+\frac{C}{v} \int_{1-a^{T}}^{r} \frac{1}{(1-t)^{39 \rho / 40} t} d t \\
\leq & \frac{1}{v} \frac{1}{\rho-\frac{\varepsilon}{2}} \frac{1}{(1-r)^{\rho-\varepsilon / 2}} \\
& +\left(1+\frac{1}{M}+\frac{1}{N}\right)\left[\frac{1}{v} \int_{0}^{r} \frac{n\left(t, \widetilde{R}_{z}\right)-n\left(0, \widetilde{R}_{z}\right)}{t} d t+\frac{n\left(0, \widetilde{R}_{z}\right)}{v} \log r\right] \\
& -\left(1+\frac{1}{M}+\frac{1}{N}\right) \frac{n\left(0, \widetilde{R}_{z}\right)}{v} \log \left(1-a^{T}\right)+\frac{C}{v} \frac{1}{\frac{39 \rho}{40}} \frac{1}{(1-r)^{39 \rho / 40}} \\
= & \frac{1}{v} \frac{1}{\rho-\frac{\varepsilon}{2}} \frac{1}{(1-r)^{\rho-\varepsilon / 2}}+\left(1+\frac{1}{M}+\frac{1}{N}\right) N\left(r, \widetilde{R}_{z}\right) \\
& -\left(1+\frac{1}{M}+\frac{1}{N}\right) \frac{n\left(0, \widetilde{R}_{z}\right)}{v} \log \left(1-a^{T}\right)+\frac{C}{v} \frac{1}{39 \rho} \frac{1}{40} \frac{1-r)^{39 \rho / 40}}{(1-} .
\end{aligned}
$$

Since $T$ is fixed, we see that $T\left(1-a^{T-1}, w\right)$ is a finite constant. 
Hence,

$$
\begin{aligned}
\frac{1}{k} \frac{1}{v} \int_{1-a^{T-1}}^{1-(1-r) / a} \frac{S(t, w)}{t} d t & \leq(2 v-1) \frac{1}{v} \int_{1-a^{T}}^{r} \frac{S\left(1-\frac{1-t}{a}\right)}{t} d t \\
\leq & \frac{1}{v} \frac{1}{\rho-\frac{\varepsilon}{2}} \frac{1}{(1-r)^{\rho-\varepsilon / 2}}+\left(1+\frac{1}{M}+\frac{1}{N}\right) N\left(r, \widetilde{R}_{z}\right) \\
& -\left(1+\frac{1}{M}+\frac{1}{N}\right) \frac{n\left(0, \widetilde{R}_{z}\right)}{v} \log \left(1-a^{T}\right) \\
& +\frac{C}{v} \frac{1}{\frac{39 \rho}{40}} \frac{1}{(1-r)^{39 \rho / 40}} .
\end{aligned}
$$

Then

$$
\begin{aligned}
\frac{1}{k v} \int_{0}^{1-(1-r) / a} \frac{S(t, w)}{t} d t \leq & \frac{1}{v} \frac{1}{\rho-\frac{\varepsilon}{2}} \frac{1}{(1-r)^{\rho-\varepsilon / 2}}+\left(1+\frac{1}{M}+\frac{1}{N}\right) N\left(r, \widetilde{R}_{z}\right) \\
& -\left(1+\frac{1}{M}+\frac{1}{N}\right) \frac{n\left(0, \widetilde{R}_{z}\right)}{v} \log \left(1-a^{T}\right) \\
& +\frac{C}{v} \frac{1}{\frac{39 \rho}{40}} \frac{1}{(1-r)^{39 \rho / 40}}+\frac{1}{k} T\left(1-a^{T-1}, w\right) .
\end{aligned}
$$

Under the condition

$$
N\left(r, \widetilde{R}_{z}\right) \leq(2 v-1) T(r, w)+O(1),
$$

we obtain

$$
\begin{aligned}
\frac{1}{k} T\left(1-\frac{1-r}{a}, w\right) \leq & \frac{1}{v\left(\rho-\frac{\varepsilon}{2}\right)} \frac{1}{(1-r)^{\rho-\varepsilon / 2}}+\left(1+\frac{1}{M}+\frac{1}{N}\right) 2(v-1) T(r, w) \\
& +C \log \left(1-a^{T}\right)+\frac{C}{v} \frac{1}{\frac{39 \rho}{40}} \frac{1}{(1-r)^{39 \rho / 40}}+C,
\end{aligned}
$$

where $C$ is a constant.

Dividing both sides of the above inequality by $U(1 /(1-r))=1 /(1-r)^{\rho(1 /(1-r))}$, we have

$$
\begin{aligned}
\frac{1}{k} \frac{T\left(1-\frac{1-r}{a}, w\right)}{U\left(\frac{1}{1-r}\right)} \leq & \frac{1}{v\left(\rho-\frac{\varepsilon}{2}\right)} \frac{(1-r)^{\rho(1 /(1-r))}}{(1-r)^{\rho-\varepsilon / 2}} \\
& +\left(1+\frac{1}{M}+\frac{1}{N}\right) 2(v-1) \frac{T(r, w)}{U\left(\frac{1}{1-r}\right)} \\
& +C \frac{\log \left(1-a^{T}\right)}{\left(\frac{1}{1-r}\right)^{\rho(1 /(1-r))}}+\frac{C}{v} \frac{1}{\frac{39 \rho}{40}} \frac{(1-r)^{\rho(1 /(1-r))}}{(1-r)^{39 \rho / 40}} \\
& +\frac{C}{\left(\frac{1}{1-r}\right)^{\rho(1 /(1-r))}}
\end{aligned}
$$


We note that

$$
\frac{T\left(1-\frac{1-r}{a}, w\right)}{U\left(\frac{1}{1-r}\right)}=\frac{T\left(1-\frac{1-r}{a}, w\right)}{U\left(\frac{a}{1-r}\right)} \frac{U\left(\frac{a}{1-r}\right)}{U\left(\frac{1}{1-r}\right)} .
$$

In view of the properties of the $U(1 /(1-r))$, we obtain

$$
\begin{aligned}
\limsup _{r \rightarrow 1-} \frac{T\left(1-\frac{1-r}{a}, w\right)}{U\left(\frac{1}{1-r}\right)} & \geq \limsup _{r \rightarrow 1-} \frac{T\left(1-\frac{1-r}{a}, w\right)}{U\left(\frac{a}{1-r}\right)} \liminf _{r \rightarrow 1-} \frac{U\left(\frac{a}{1-r}\right)}{U\left(\frac{1}{1-r}\right)} \\
& =\limsup _{r \rightarrow 1-} \frac{T\left(1-\frac{1-r}{a}, w\right)}{U\left(\frac{a}{1-r}\right)} \lim _{r \rightarrow 1-} \frac{U\left(\frac{a}{1-r}\right)}{U\left(\frac{1}{1-r}\right)} \\
& =a^{\rho} .
\end{aligned}
$$

Letting $r \rightarrow 1-$ in (2.2),

$$
(2 v+1) \frac{1}{k} a^{\rho} \leq\left(1+\frac{1}{M}+\frac{1}{N}\right) 2(v-1) .
$$

That is,

$$
2 v+1 \leq\left(1+\frac{1}{M}+\frac{1}{N}\right) 2(v-1) k a^{-\rho} .
$$

Letting $a \rightarrow 1-, M \rightarrow+\infty$, and $N \rightarrow+\infty$, we obtain

$$
k \geq \frac{2 v+1}{2 v-2}
$$

This contradicts part (i) of the claim, and the lemma is proved.

\section{Proof of the theorem}

Proof. Choose $\varepsilon_{n}=\rho / 2 n$ and $R_{n}=1-1 / 2^{n}$.

In view of Lemma 2.2, there exist

$$
a_{n} \in\left(1-\frac{1}{n}, 1\right), \quad m_{n}=\left\lfloor\frac{2 \pi}{1-a_{n}}\right\rfloor, \quad p_{n}, \quad q_{n}, \quad \theta_{q_{n}}=\frac{2 \pi\left(q_{n}\right)+1}{m_{n}},
$$

and

$$
\Omega_{p_{n} q_{n}}=\left\{1-a_{n}^{p_{n}} \leq|z| \leq 1-a_{n}^{p_{n}+2}\right\} \cap\left\{\left|\arg z-\theta_{q_{n}}\right| \leq \frac{2 \pi}{m_{n}}\right\}, \quad n=1,2, \ldots
$$

Let

$$
\theta_{n}=\theta_{q_{n}}, \quad z_{n}=\left(1-a_{n}^{p_{n}}\right) e^{i \theta_{n}}
$$

Then

$$
1>r_{n}=\left|z_{n}\right|=1-a_{n}^{p_{n}}>R_{n}=1-\frac{1}{2^{n}} \rightarrow 1-\quad \text { as } n \rightarrow+\infty \text { and } \lim _{n \rightarrow+\infty} a_{n}^{p_{n}}=0 .
$$


Set

$$
\begin{aligned}
B_{n} & =\left[\left(1-a_{n}^{p_{n}+2}\right)-\left(1-a_{n}^{p_{n}}\right)\right]+\left(1-a_{n}^{p_{n}+2}\right) \frac{2 \pi}{m_{n}} \\
& \leq\left[\left(1-a_{n}^{2 p_{n}}\right)-\left(1-a_{n}^{p_{n}}\right)\right]+\left(1-a_{n}^{2 p_{n}}\right) \frac{2 \pi}{m_{n}} \\
& =\left(1-a_{n}^{p_{n}}\right) a_{n}^{p_{n}}+\left(1-a_{n}^{p_{n}}\right)\left(1+a_{n}^{p_{n}}\right) \frac{2 \pi}{m_{n}} \\
& \leq\left(1-a_{n}^{p_{n}}\right)\left[a_{n}^{p_{n}}+\left(1+a_{n}^{p_{n}}\right) \frac{2 \pi}{m_{n}}\right] .
\end{aligned}
$$

Take

$$
\sigma_{n}=a_{n}^{p_{n}}+\left(1+a_{n}^{p_{n}}\right) \frac{2 \pi}{m_{n}} ;
$$

then

$$
\sigma_{n} \rightarrow 0, \quad n \rightarrow+\infty
$$

Put

$$
\Gamma_{n}=\left\{\left|z-z_{n}\right|<r_{n} \sigma_{n}\right\} .
$$

Then

$$
\Omega_{p_{n} q_{n}} \subset \Gamma_{n} .
$$

In view of Lemma 2.2, for each $n$, the function $w(z)$ takes every complex number at least $a_{n}^{-p_{n}\left(\rho-\varepsilon_{n}\right)}=\left(1-r_{n}\right)^{-\rho+\varepsilon_{n}}$ times in $\Gamma_{n} \cap \Delta$, except for those complex numbers contained in the union of $2 \nu$ spherical disks each with radius $a_{n}^{p_{n} \rho / 40}=\left(1-r_{n}\right)^{\rho / 40}$. Theorem 1.2 is proved.

\title{
References
}

[1] W. K. Hayman, Meromorphic Functions (Clarendon Press, Oxford, 1964).

[2] H. Selberg, 'Algebroide Funktionen und Umkehrfunktionen Abelscher Integrale', Avh. Nor. Vidensk. Akad. Oslo 8 (1934), 1-72.

[3] D. C. Sun, 'Filling disks of algebroid functions', Chin. Ann. Math., Ser. A 16 (1995), 238-243.

[4] E. Ullrich, 'Über den Einfluss der Verzweigtheit einer Algebroide auf ihre Wertverteilung', J. reine angew. Math. 169 (1931), 198-220.

[5] G. Valiron, 'Sur la derivée des fonctions algébroïdes', Bull. Sci. Math. 59 (1931), 17-39.

\author{
ZU-XING XUAN, Department of Mathematical Sciences, Tsinghua University, \\ Beijing, 100084, PR China \\ and \\ Basic Department, Beijing Union University, No. 97 Bei Si Huan Dong Road, \\ Chaoyang District, Beijing, 100101, PR China \\ e-mail: xuanzuxing@ss.buaa.edu.cn
}

